\title{
Association of MDM2 promoter T309G polymorphism with oral cancer risk: A meta-analysis of 3,536 subjects
}

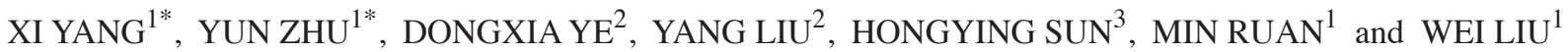 \\ ${ }^{1}$ Department of Oral and Maxillofacial-Head and Neck Oncology; ${ }^{2}$ Shanghai Key Laboratory of Stomatology, \\ Ninth People's Hospital, Shanghai Jiao Tong University School of Medicine, Shanghai 200011; \\ ${ }^{3}$ Department of Stomatology, Huashan Hospital, Fudan University, Shanghai 200040, P.R. China
}

Received March 6, 2015; Accepted April 7, 2016

DOI: $10.3892 / \operatorname{mco} .2016 .868$

\begin{abstract}
The mouse double minute 2 (MDM2) gene is an important regulator of the p53 suppressor gene. To date, evidence concerning the association of the MDM2 single nucleotide polymorphism (SNP) 309T >G (rs2279744) with the risk of developing oral squamous cell carcinoma (OSCC) remains controversial. Therefore, a meta-analysis of all the eligible studies was performed, in order to derive a more precise estimation of this association. Odds ratios (ORs) with $95 \%$ confidence intervals (CIs) were used to assess the degree of association in 5 previous studies, including a total of 1,369 OSCC cases and 2,167 controls. The overall analysis revealed a significant association between MDM2 SNP309 and OSCC risk in the heterozygote (TG vs. TT: $\mathrm{OR}=0.81 ; 95 \% \mathrm{CI}: 0.68-0.96 ; \mathrm{P}=0.02$ ) and dominant models $(\mathrm{TG}+\mathrm{GG}$ vs. TT: $\mathrm{OR}=0.82 ; 95 \% \mathrm{CI}$ : 0.69-0.97; $\mathrm{P}=0.02$ ). The subgroup analysis based on the source of the controls revealed a significant association between population-based controls and the heterozygote model (TG vs. TT: $\mathrm{OR}=0.75 ; 95 \% \mathrm{CI}: 0.62-0.91 ; \mathrm{P}=0.004)$, dominant model (TG+GG vs. TT: OR=0.76; 95\% CI: $0.63-0.91 ; \mathrm{P}=0.003)$ and allele comparison $(\mathrm{G}$ vs. $\mathrm{T}$ : $\mathrm{OR}=0.89$; 95\% CI: 0.79-0.99; $\mathrm{P}=0.04$ ). Importantly, no evidence of publication bias or obvious heterogeneity were observed in the meta-analysis. The results of the present study demonstrated a decreased risk of developing OSCC for the MDM2 SNP309 group, suggesting MDM2 SNP309 may be a protection-associated genetic variation for OSCC. Additional well-designed
\end{abstract}

Correspondence to: Dr Min Ruan or Wei Liu, Department of Oral and Maxillofacial-Head and Neck Oncology, Ninth People's Hospital, Shanghai Jiao Tong University School of Medicine, No. 18 Zhi Zao Ju Road 833, Shanghai 200011, P.R. China

E-mail: doctorruanmin@hotmail.com

E-mail: liuweb@hotmail.com

*Contributed equally

Key words: mouse double minute 2, polymorphism, single-nucleotide polymorphism, oral cancer, meta-analysis studies, with larger sample sizes, are required to further elucidate this association.

\section{Introduction}

Oral cancer is one of the most common cancers worldwide, with an unclear pathogenesis; the oral squamous cell carcinoma (OSCC) subtype accounts for $>90 \%$ of all oral cancers (1). While increasing evidence suggests that environmental factors, as well as chemical carcinogens (including tobacco and alcohol) are likely etiological factors that contribute to the development of OSCC, only a small minority of individuals exposed to these carcinogens will subsequently develop head and neck SCC (2,3). Oral carcinogenesis is widely recognized as a stepwise process, and the involvement of genetic alterations and host polymorphisms may be important to its development (4). The identification of a predictive model of risk polymorphisms may facilitate early diagnosis and the understanding of disease progression in a subset of cancer patients (4).

The human mouse double minute 2 (MDM2) gene is an important negative regulator of the p53 suppressor gene, promoting the degradation of p53 through its E3 ubiquitin ligase activity (5). A functional single-nucleotide polymorphism (SNP), rs2279744, is located at nucleotide 309 in the first intron of the MDM2 promoter region, consisting of a change from $\mathrm{T}$ to $\mathrm{G}$. This mutation has been named SNP309 (6). SNP 309T $>\mathrm{G}$ is known to enhance the binding affinity of the transcriptional activator SP1, contributing to increased expression of MDM2 and subsequent attenuation of the p53 tumor suppressor pathway (6). MDM2 SNP309 and its association with cancer susceptibility has been identified and extensively investigated in a number of cancer types $(7,8)$.

To date, numerous previous studies have investigated the association between MDM2 SNP309 and OSCC risk (9-15). However, the results remain inconsistent and ambiguous, partly due to the relatively small sample sizes of the independent studies and sampling effects. Meta-analyses allow stronger conclusions for identifying certain models of risk markers, which may help with screening, early diagnosis and/or therapy in the clinical setting (16-18). Therefore, a meta-analysis of all eligible studies was performed, in order to achieve a more precise estimation of this association, and to investigate the source of heterogeneity and any potential bias in this method. 
Table I. Study characteristics of the meta-analysis.

\begin{tabular}{|c|c|c|c|c|c|c|c|c|c|c|c|c|c|}
\hline \multirow{2}{*}{$\begin{array}{l}\text { Author, } \\
\text { year (Refs.) }\end{array}$} & \multirow[b]{2}{*}{ Country } & \multirow[b]{2}{*}{ Ethnicity } & \multirow{2}{*}{$\begin{array}{c}\text { Source } \\
\text { of controls }\end{array}$} & \multirow[b]{2}{*}{ Genotyping } & \multirow{2}{*}{$\begin{array}{c}\text { HWE } \\
\text { of controls }\end{array}$} & \multicolumn{2}{|c|}{ Sample (n) } & \multicolumn{3}{|c|}{ Cases } & \multicolumn{3}{|c|}{ Controls } \\
\hline & & & & & & Cases & Controls & $\mathrm{TT}$ & TG & GG & $\mathrm{TT}$ & TG & GG \\
\hline $\begin{array}{l}\text { Tu et al, } \\
2008 \text { (9) }\end{array}$ & Taiwan & Asian & Hospital & PCR-RFLP & 0.582 & 189 & 116 & 44 & 93 & 52 & 29 & 55 & 32 \\
\hline $\begin{array}{l}\text { Huang et al, } \\
2009(10)\end{array}$ & Taiwan & Asian & Population & MALDI-TOF & 0.286 & 351 & 1272 & 80 & 176 & 95 & 274 & 653 & 345 \\
\hline $\begin{array}{l}\text { Misra et al, } \\
2009 \text { (11) }\end{array}$ & India & Asian & Population & PCR-RFLP & 0.042 & 297 & 328 & 70 & 147 & 80 & 59 & 181 & 88 \\
\hline $\begin{array}{l}\text { Hamid et al, } \\
2009(12)\end{array}$ & Malaysia & Asian & Hospital & PCR-RFLP & 0.997 & 207 & 116 & 48 & 104 & 55 & 30 & 58 & 28 \\
\hline $\begin{array}{l}\text { Chen et al, } \\
2009 \text { (13) }\end{array}$ & USA & Caucasian & Population & PCR-RFLP & 0.835 & 325 & 335 & 146 & 132 & 47 & 112 & 165 & 58 \\
\hline
\end{tabular}

HWE, Hardy-Weinberg equilibrium; PCR-RFLP, polymerase chain reaction-restriction fragment length polymorphism; MALDI-TOF, matrix-assisted laser desorption/ionization time-of-flight.

\section{Materials and methods}

Literature search strategy. The electronic databases PubMed, Web of Science, Embase and China National Knowledge Infrastructure were searched for relevant publications to be included in the present meta-analysis, without restriction on language and publication year (until January 10, 2015). The following search terms were used: ('murine double minute 2' OR 'MDM2') AND ('polymorphisms' OR 'variants') AND ('carcinoma' OR 'cancer' OR 'malignancy' OR 'neoplasm' OR 'tumour' OR 'tumor') AND ('oral'). Relevant articles were reviewed to evaluate their appropriateness for inclusion in the meta-analysis. Additional relevant publications were identified through the references cited in the articles selected or review articles on this topic.

Inclusion and exclusion criteria. The inclusion criteria for eligible articles were as follows: i) Case-control studies on humans; ii) evaluation of MDM2 rs2279744 or 309T $>\mathrm{G}$ polymorphism and OSCC risk; iii) sufficient genotype data to estimate an odds ratio (OR) and $95 \%$ confidence interval (CI); and iv) histologically confirmed diagnosis of OSCC. The exclusion criteria were as follows: i) Not a case-control study; ii) no usable data reported; and iii) duplicate or overlapping data.

Data extraction. According to the selection criteria, all relevant crude data were extracted from each eligible article independently by two researchers, and the inconsistencies were discussed until a consensus was obtained. The following items were extracted from each article: First author name, year of publication, country of origin, ethnicity, genotyping method, source of control (population- or hospital-based), number of cases and controls, characteristics of cancer cases and controls, and genotype frequencies for cases and controls.

Statistical analysis. The Hardy-Weinberg equilibrium (HWE) of the control group in each study was measured using either

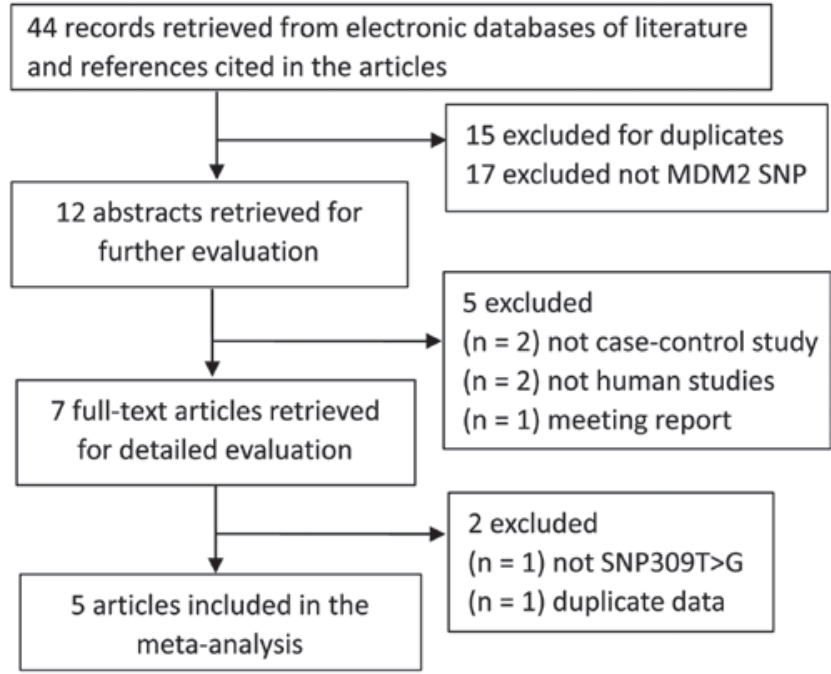

Figure 1. Flow diagram of the study selection process. MDM2, mouse double minute 2; SNP, single-nucleotide polymorphism.

the $\chi^{2}$ or Fisher's exact test. The degree of association between MDM2 SNP309 and OSCC risk was determined using the OR with 95\% CI. In the overall and subgroup meta-analysis, pooled ORs and 95\% CIs for the heterozygote (TG vs. TT), homozygote (GG vs. TT), dominant (TG+GG vs. TT) and recessive models (GG vs. TG+TT) were calculated. The allele comparison ( $\mathrm{G}$ vs. $\mathrm{T}$ ) was conducted as an additive mode. The statistical significance of the pooled OR was evaluated using the Z-test, and the heterogeneity of the ORs was tested by $\chi^{2}$-based Q-test and $\mathrm{I}^{2}$ statistics. If the result of heterogeneity test was $\mathrm{P}>0.1$, ORs were pooled according to the fixed-effects model (Mantel-Haenszel). Otherwise, the random-effects model (DerSimonian and Laird) was used. Furthermore, the Egger's test and Begg's funnel plot were used to estimate the potential publication bias. All statistical analyses were performed with the software Stata 10.0 (StataCorp LP, College Station, TX, USA) 
Table II. Summary of pooled odds ratios with $95 \%$ confidence intervals in the meta-analysis.

\begin{tabular}{|c|c|c|c|c|c|}
\hline \multirow[b]{2}{*}{ Group (no. of cases/controls) } & \multicolumn{3}{|c|}{ Test of association } & \multicolumn{2}{|c|}{ Test of heterogeneity } \\
\hline & Genotype & OR $(95 \% \mathrm{CI})$ & P-value & $\mathrm{I}^{2}(\%)$ & P-value \\
\hline \multirow{5}{*}{$\begin{array}{l}\text { Overall } \\
(1,369 / 2,167)\end{array}$} & TG vs. TT & $0.81(0.68-0.96)$ & 0.02 & 38 & 0.17 \\
\hline & GG vs. TT & $0.86(0.70-1.06)$ & 0.17 & 2 & 0.39 \\
\hline & TG+GG vs. TT & $0.82(0.69-0.97)$ & 0.02 & 45 & 0.13 \\
\hline & GG vs. TG+TT & $0.98(0.83-1.16)$ & 0.80 & 0 & 0.88 \\
\hline & G vs. T & $0.92(0.83-1.02)$ & 0.11 & 34 & 0.20 \\
\hline \multicolumn{6}{|l|}{ Subgroup } \\
\hline \multirow{5}{*}{$\begin{array}{l}\text { Population-based controls } \\
(973 / 1,935)\end{array}$} & TG vs. TT & $0.75(0.62-0.91)$ & 0.004 & 41 & 0.18 \\
\hline & GG vs. TT & $0.80(0.63-1.01)$ & 0.06 & 6 & 0.35 \\
\hline & TG+GG vs. TT & $0.76(0.63-0.91)$ & 0.003 & 47 & 0.15 \\
\hline & GG vs. TG+TT & $0.96(0.79-1.16)$ & 0.65 & 0 & 0.67 \\
\hline & G vs. T & $0.89(0.79-0.99)$ & 0.04 & 48 & 0.15 \\
\hline \multirow{5}{*}{$\begin{array}{l}\text { Asian } \\
(1,044 / 1,832)\end{array}$} & TG vs. TT & $0.90(0.73-1.11)$ & 0.32 & 8 & 0.41 \\
\hline & GG vs. TT & $0.94(0.75-1.19)$ & 0.61 & 2 & 0.66 \\
\hline & TG+GG vs. TT & $0.94(0.75-1.19)$ & 0.37 & 0 & 0.42 \\
\hline & GG vs. TG+TT & $1.02(0.85-1.22)$ & 0.87 & 0 & 0.98 \\
\hline & G vs. T & $0.98(0.87-1.09)$ & 0.67 & 0 & 0.73 \\
\hline
\end{tabular}

Bold print indicates significant results. All models for the test of heterogeneity were fixed. OR, odds ratio; CI, confidence interval.

and Review Manager 5.0 (Cochrane Informatics and Knowledge Management, London, UK), using two-sided P-values.

\section{Results}

Study characteristics. Of 44 potentially relevant articles, 37 were gradually excluded (Fig. 1), and 7 full-text articles were selected for detailed evaluation from our search of the published literature. During the extraction of data, one study by Canova et al (14) that was not relevant to MDM2 309T>G (rs2279744) polymorphism was excluded; in addition, one study by Wang et al (15) that included overlapping data from the authors' colleague was excluded according to the inclusion and exclusion criteria. Therefore, 5 eligible articles, including a total of 1,369 OSCC cases and 2,167 controls, were ultimately included in the final meta-analysis (Fig. 1). The characteristics of the eligible studies are summarized in Table I. All were case-control studies, with Asian and Caucasian subjects. There were 2 studies with hospital-based and 3 studies with population-based controls. A classic polymerase chain reaction-restriction fragment length polymorphism (PCR-RFLP) assay was performed in 4 of the 5 studies. The detailed variant genotype distributions of MDM2 SNP309 for OSCC cases and controls in the individual studies are listed in Table I.

Meta-analysis results. The associations between MDM2 SNP309 variants and OSCC risk are summarized in Table II. In the overall analysis, the heterozygote (TG vs. TT: $\mathrm{OR}=0.81 ; 95 \% \mathrm{CI}: 0.68-0.96 ; \mathrm{P}=0.02)$ and dominant genetic models (TG+GG vs. TT: OR=0.82; 95\% CI: 0.69-0.97; $\mathrm{P}=0.02$ ) of MDM2 SNP309 were significantly associated with OSCC risk, while the results of homozygote and recessive models and allele comparison were not significant. Forest plots for the meta-analysis of the two significant genetic models are shown in Fig. 2. In the subgroup analysis based on the source of the controls, significant associations were observed in the population-based controls among the heterozygote (TG vs. TT: $\mathrm{OR}=0.75 ; 95 \% \mathrm{CI}: 0.62-0.91 ; \mathrm{P}=0.004)$ and dominant models (TG+GG vs. TT: OR=0.76; 95\% CI: 0.63-0.91; $\mathrm{P}=0.003$ ), and allele comparison ( $\mathrm{G}$ vs. $\mathrm{T}$ : $\mathrm{OR}=0.89 ; 95 \% \mathrm{CI}$ : 0.79-0.99; $\mathrm{P}=0.04$ ). In the ethnicity subgroup analysis, no significant association in Asians was found among any genetic model. As there was only one Caucasian study, the data of the Caucasian stratified analysis are not shown (Table II).

Test of heterogeneity. As seen in Table II, there was no evidence of heterogeneity in any of the genetic models (all $\mathrm{P}>0.1$ for heterogeneity, Q-test). Therefore, a summary of ORs was analyzed with the fixed-effects model (Mantel-Haenszel).

Publication bias. Publication bias was evaluated using the Begg's funnel plot and Egger's test. As shown in Fig. 3, the shape of Begg's funnel plots appeared to be largely symmetrical in both the heterozygote and dominant models; in addition, the Egger's test revealed no significant publication bias $(\mathrm{P}=0.433$ and $\mathrm{P}=0.412$, respectively).

\section{Discussion}

MDM2 is an important regulator of the p53 suppressor gene, and the associations between the MDM2 SNP309 mutation and cancer susceptibility have been evaluated in a number of cancer types $(7,8)$. Previously published studies on the 

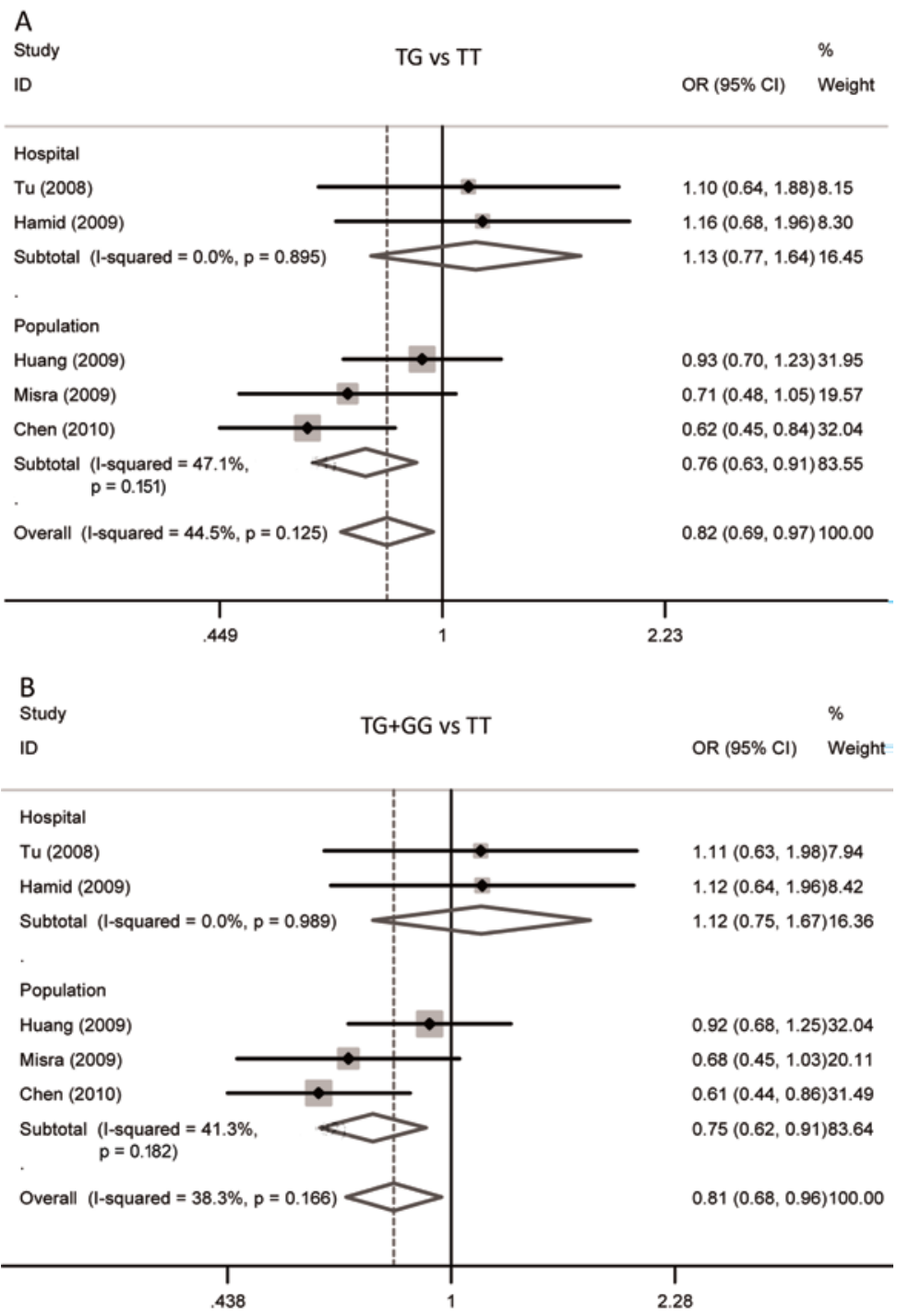

Figure 2. Forest plots of the meta-analysis. (A) Heterozygote genetic model (TG vs. TT) and (B) dominant genetic model (TG+GG vs. TT). OR, odds ratio; $\mathrm{CI}$, confidence interval.
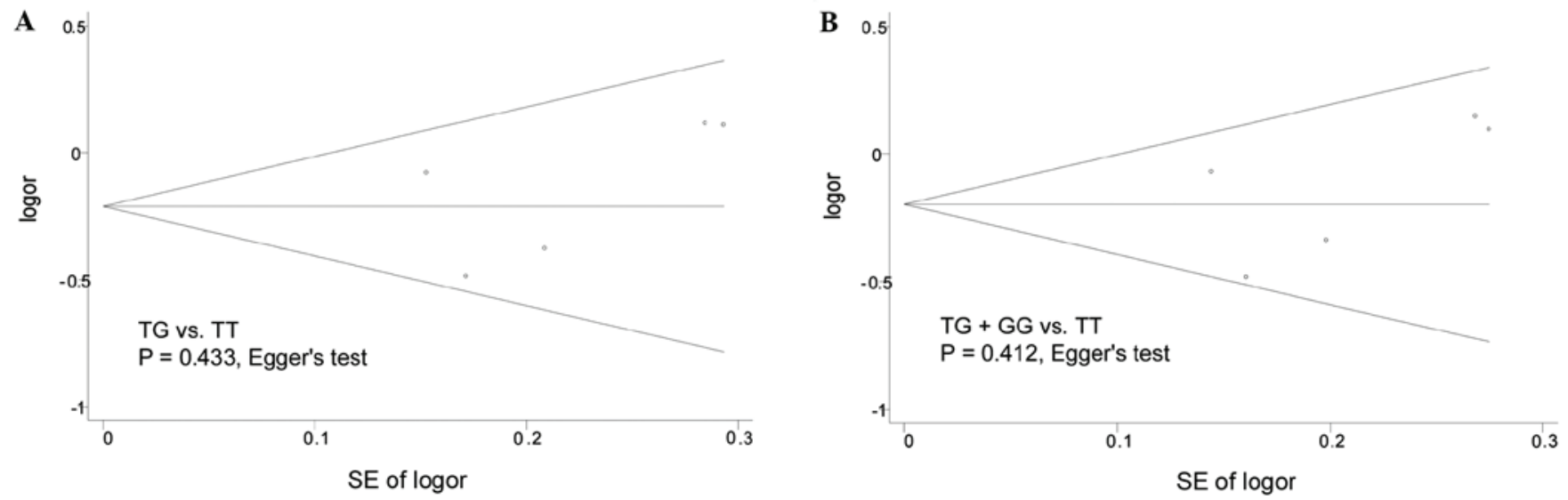

Figure 3. Begg's funnel plots and Egger's test with pseudo 95\% confidence limits. (A) Heterozygote genetic model (TG vs. TT) and (B) dominant genetic model (TG+GG vs. TT). SE, standard error.

association of this SNP with OSCC risk have generated variable results (9-13). The aim of the present study was to obtain a more precise estimation of the association between
MDM2 SNP309 and OSCC susceptibility by performing a meta-analysis that included all eligible case-control studies published to date. 
Five independent studies investigating the genetic effects of MDM2 SNP309T>G on OSCC risk (9-13) were included in this meta-analysis. Among these, 1 study demonstrated that MDM2 SNP309T $>\mathrm{G}$ was associated with a reduced risk of OSCC (13), while the remaining 4 studies provided no evidence of an association between this SNP and OSCC risk (9-12). A meta-analysis of the 5 studies, including 1,369 cases and 2,167 controls, was performed to investigate the overall effects of MDM2 SNP309 on OSCC risk. The overall analysis revealed that the MDM2 SNP309 heterozygote and dominant models were inversely associated with OSCC risk, with no significant heterogeneity. These results suggest that the MDM2 SNP309T $>$ G may be a protective factor for OSCC, which is consistent with previous analyses performed on prostate and ovarian cancers (19-21).

Although a number of previous meta-analyses have demonstrated that MDM2 SNP309T $>$ G may be a risk factor for several cancer types, including cervical, gastric and hepatocellular cancers $(7,8)$, there are also analyses that provide evidence regarding its protective role in prostate and ovarian cancers (19-21), in addition to the present analysis for oral cancer. Cancer development is an extremely complex process, and different cancer types exhibit genetic heterogeneity. Although MDM2 SNP309 is a plausible carcinogenic hereditary factor in the MDM2-p53 pathway, MDM2 is activated in response to a variety of oncogenic pathways that are independent of p53 (22). Furthermore, Francis et al (23) reported that MDM2 has no significant role in stabilizing the expression of p53, and remarked on the presence of unknown interactors that sequester p53 and lead to its aberrant accumulation. Additionally, there is evidence that decreased MDM2 expression is associated with a worse prognosis of head and neck carcinomas (24). Taken together, these findings suggest that MDM2 may have tumor suppressor functions under certain conditions. Therefore, gene-gene and gene-environment interactions regulate carcinogenesis, and the presence of other causal factors as yet unidentified demonstrate an involvement of MDM2 SNP309 in OSCC development.

Consistent with the results of the overall analysis, the subgroup analysis revealed that MDM2 SNP309 significantly reduced OSCC risk in the population-based controls, while no significant association was found in hospital-based controls. A possible explanation is that the hospital-based controls may represent a sample of an ill-defined reference population rather than the general population, and inherent selection bias may therefore not be completely excluded. Thus, use of more appropriate population-recruited control subjects may be crucial for reducing study bias. Additionally, the ethnicity subgroup analysis demonstrated a significant association between MDM2 SNP309 and OSCC risk among Caucasians, but not Asians, suggesting that SNP309 may be an ethnicity-dependent factor associated with OSCC risk.

Due to several limitations of the present meta-analysis, the results should be interpreted with caution. First, the number of the published studies eligible for inclusion and the pooled sample size of the independent studies were relatively small in the overall and subgroup analyses. In particular, only one relevant study with Caucasian patients was identified, and it is possible that some relevant unpublished studies were overlooked. Second, the effect of the confounding factors, resulting from gene-gene (such as the p53 pathway) and gene-environment (such as tobacco and alcohol consumption) interactions were not evaluated in the present study due to data limitations. Thus, in order to obtain a more precise analysis of the effect of MDM2 SNP309 mutation on OSCC risk, additional, improved studies with larger sample sizes and diverse ethnicities, particularly Caucasians and Africans, are required. However, despite these limitations, the present meta-analysis has some strengths. First, a systematic review of the association between MDM2 SNP309 and OSCC risk is statistically more powerful compared with any independent study. Second, the quality of the eligible studies included in the present meta-analysis was acceptable, and there was no evidence of publication bias or obvious outcome heterogeneity.

In conclusion, the present meta-analysis achieved a more precise evaluation of the association between MDM2 SNP309 and OSCC risk compared with independent studies. The results of the meta-analysis indicate that the MDM2 SNP309 mutation may serve as a protective factor in OSCC; however, this is only a preliminary analysis and the results presented herein should be interpreted with caution. Further well-designed and larger studies are required to elucidate the association between MDM2 SNP309 and OSCC risk.

\section{Acknowledgements}

The present study was supported by grants from the National Natural Science Foundation of China (81472517, 81102049, 81302358 and 81202132), the Shanghai Pujiang Talent Program (15PJD024), the Shanghai Education Commission Project of Young Teachers (ZZjdyx13104) and the Shanghai Natural Science Foundation (13ZR1457100).

\section{References}

1. Krishna Rao SV, Mejia G, Roberts-Thomson K and Logan R: Epidemiology of oral cancer in Asia in the past decade-an update (2000-2012). Asian Pac J Cancer Prev 14: 5567-5577, 2013.

2. Petersen PE: Oral cancer prevention and control-the approach of the World Health Organization. Oral Oncol 45: 454-460, 2009.

3. Zini A, Czerninski R and Sgan-Cohen HD: Oral cancer over four decades: Epidemiology, trends, histology, and survival by anatomical sites. J Oral Pathol Med 39: 299-305, 2010.

4. Liao G, Wang Y, Zhou YQ, Li TW, Zeng DQ, Zeng X, Li J, Dan HX and Chen QM: Host genetic susceptibility to oral cancer: Evidence from meta-analyses and pooled analyses. Oral Dis 20: 644-649, 2014.

5. Bond GL, Hu W, Bond EE, Robins H, Lutzker SG, Arva NC, Bargonetti J, Bartel F, Taubert H, Wuerl P, et al : A single nucleotide polymorphism in the MDM2 promoter attenuates the p53 tumor suppressor pathway and accelerates tumor formation in humans. Cell 119: 591-602, 2004.

6. Bond GL, Hu W and Levine A: A single nucleotide polymorphism in the MDM2 gene: From a molecular and cellular explanation to clinical effect. Cancer Res 65: 5481-5484, 2005.

7. Wo X, Han D, Sun H, Liu Y, Meng X, Bai J, Chen F, Yu Y, Jin Y and Fu S: MDM2 SNP309 contributes to tumor susceptibility: A meta-analysis. J Genet Genomics 38: 341-350, 2011.

8. Chen B, Cao L, Hu KW, Zhang JW, Meng XL and Xiong MM: MDM2 SNP309 is an ethnicity-dependent risk factor for digestive tract cancers. Tumour Biol 35: 3431-3438, 2014.

9. Tu HF, Chen HW, Kao SY, Lin SC, Liu CJ and Chang KW: MDM2 SNP 309 and p53 codon 72 polymorphisms are associated with the outcome of oral carcinoma patients receiving postoperative irradiation. Radiother Oncol 87: 243-252, 2008.

10. Huang SF, Chen IH, Liao CT, Wang HM, Liou SH and Hsieh LL: Combined effects of MDM2 SNP 309 and p53 mutation on oral squamous cell carcinomas associated with areca quid chewing. Oral Oncol 45: 16-22, 2009. 
11. Misra C, Majumder M, Bajaj S, Ghosh S, Roy B and Roychoudhury S: Polymorphisms at p53, p73, and MDM2 loci modulate the risk of tobacco associated leukoplakia and oral cancer. Mol Carcinog 48: 790-800, 2009.

12. Hamid S, Yang YH, Peng KN, Ismail SM, Zain RB, Lim KP Wan Mustafa WM, Abraham MT, Teo SH and Cheong SC: MDM2 SNP309 does not confer an increased risk to oral squamous cell carcinoma but may modulate the age of disease onset. Oral Oncol 45: 496-500, 2009.

13. Chen X, Sturgis EM, Lei D, Dahlstrom K, Wei Q and Li G: Human papillomavirus seropositivity synergizes with MDM2 variants to increase the risk of oral squamous cell carcinoma. Cancer Res 70: 7199-7208, 2010

14. Canova C, Hashibe M, Simonato L, Nelis M, Metspalu A, Lagiou P, Trichopoulos D, Ahrens W, Pigeot I, Merletti F, et al : Genetic associations of 115 polymorphisms with cancers of the upper aerodigestive tract across 10 European countries: The ARCAGE project. Cancer Res 69: 2956-2965, 2009.

15. Wang Z, Sturgis EM, Zhang Y, Huang Z, Zhou Q, Wei Q and Li G: Combined p53-related genetic variants together with HPV infection increase oral cancer risk. Int J Cancer 131: E251-E258, 2012.

16. Zhang Y, Li S, Xiao HQ, Hu ZX, Xu YC and Huang Q: Vascular endothelial growth factor gene polymorphisms and renal cell carcinoma: A systematic review and meta-analysis. Oncol Lett 6: 1068-1078, 2013.

17. Wang JG, Zhang Y and Xiao TL: Quantitative analysis of the association between CRP rs2808630 and rs1417938 polymorphisms and cancer risk. Oncol Lett 9: 994-998, 2015.
18. Jiang LL and Ruan LW: Association between FOXP3 promoter polymorphisms and cancer risk: A meta-analysis. Oncol Lett 8: 2795-2799, 2014

19. Chen T, Yi SH, Liu XY and Liu ZG: Meta-analysis of associations between the MDM2-T309G polymorphism and prostate cancer risk. Asian Pac J Cancer Prev 13: 4327-4330, 2012.

20. Liu G, Jiang D, Shen S and Yu L: Murine double minute 2 promoter SNP309 polymorphism and prostate cancer risk: A meta-analysis. Int J Urol 19: 914-920, 2012.

21. Ma YY, Guan TP, Yao HB, Yu S, Chen LG, Xia YJ, He XJ, Wang HJ, Jiang XT and Tao HQ: The MDM2 309T>G polymorphism and ovarian cancer risk: A meta-analysis of 1534 cases and 2211 controls. PLoS One 8: e55019, 2013.

22. Manfredi JJ: The Mdm2-p53 relationship evolves: Mdm2 swings both ways as an oncogene and a tumor suppressor. Genes Dev 24: 1580-1589, 2010.

23. Francis G, Dileep Kumar U, Nalinakumari KR, Jayasree K and Kannan S: Accumulation of inactive p53 protein in oral squamous cell carcinoma: Stabilization by protein interaction. Eur J Oral Sci 121: 21-28, 2013.

24. Millon R, Muller D, Schultz I, Salvi R, Ghnassia JP, Frebourg T, Wasylyk B and Abecassis J: Loss of MDM2 expression in human head and neck squamous cell carcinomas and clinical significance. Oral Oncol 37: 620-631, 2001. 